\title{
ORAL PROPHYLAXIS IN ITS RELATION TO PYORRHEA AND ITS TREATMENT.
}

\author{
The Work Relative to the Entamoeba in This Paper is the \\ Product of the Research Commission of the National \\ Dental Association.
}

By Arthur T. Henrici, M. D., and the Author of this Paper, Thomas B.

Hartzell, M. D., D. D. S.

(Read before the Chicago Dental Sucjety, April 20, 1915.)

$\mathrm{P}$ YORRHEA alveolaris begins in the gum margins. The writer of this article voiced this thought in a paper read before the Northeastern Dental Association in 1911.* Perhaps no more conclusive argument can be adduced on this point than the fact that every dentist has noticed that the removal of infected teeth results ninety-nine times out of a hundred in cessation of all inflammatory symptoms. The second corroborative fact is that removal of the infected porous root surface usually checks all types of interstitial gingivitis quickly if the work be accurately done, but if the tooth's surface be neglected and dirty, gingivitis recurs.

In the observation of more than two thousand cases, the author has found no exception to this general rule, except in cases of acute diffused nephritis, diabetes-mellitus, and certain types of drug poisoning. The above observation was published in the Canadian Dental Journal, June 5, 1912.

That gingival inflammation is the orioinating point from which pyorrhoeal inflammations continue has been observed by many writers. A close study of the

*Published in the Dental Cosmos, 1911. pictures of transverse section of both human and animal jaws by Talbot, (*1) and human jaws by Hopewell Smith (*2) and Znamensky (*3) of the University of Moscow, all show the deepest inflammation in the gingivae and the masses of leucocytes progressively less as we recede from the gingival margin. This point seems to have been given little weight by Talbot and Smith in their efforts to determine the point of origin, they, becoming deeply interested in the changes going on in the deeper structures of the advanced case, while on the contrary, Znamensky emphasizes his belief that the gingival margin is the point of origin. (See lantern slides of Talbot (Fig. 1), Smith, (Fig. 2), and Znamensky (Fig. 3). Perhaps the most notable article in which a rather comprehensive series of observations has been accumulated is that of Doctor Arthur Black which was published in the Dental Cosmos, page 1219, year 1913. These ob. servations were accumulated by a group of men who worked in conjunction with him and confirmed the view already stat-

* I-Talbot-Interstitial Gingivitis

*2-Smith-Dental Cosmos, 1911.

*3-Znamensky-British Dental Journal, 1908. 
ed. In the observation and study of more than two thousand cases to date, the author has found no exception to this general rule, save those already mentioned.

Granted then that the gingival margins play an important role in this disease, our next question is, what is the explanation of this often observed fact?

Fig. 1.

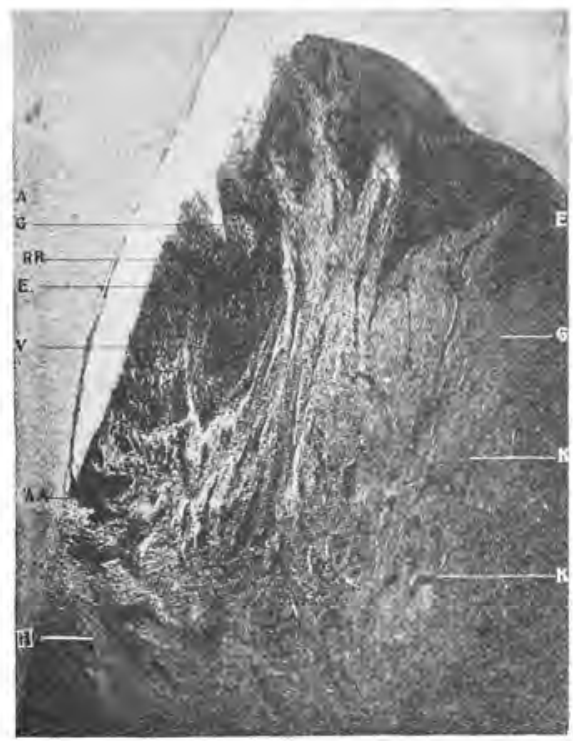

Longitudinal section of tooth and gum tissue. Chronic interstitial gingivitis. Dog. A, Enamel; E, Epithelial tissue; G, Submucous membrane; H, Periosteum; K, Capillaries; V, Violent inflammation: AA, Point of union of epithelial tissue and peridental membrane; RR, Space pocket from want of union of epithelial fold.-Talbot.

Is it because the tissues of the gingivae are less well protected, or is it because the blood supply to the gingival margins is less generous than other areas in the oral cavity. or is it because the irritating agent accumulates on the tooth's surface and constantly flows under the gingivae? Has systematic oral prophylaxis taught us anything upon which we may depend regarding this matter? Certainly there must be a rational explanation for the fact that in- flammation of the gingival margin invariably precedes deeper infection of the tissues in the neighborhood of the human teeth. What, therefore, is that explanation? The author believes the bacterial masses on the tooth surface furnish the chief irritant and the imperfectly protected gum margin permits the irritant to enter the tissues, the

Fig. 2.

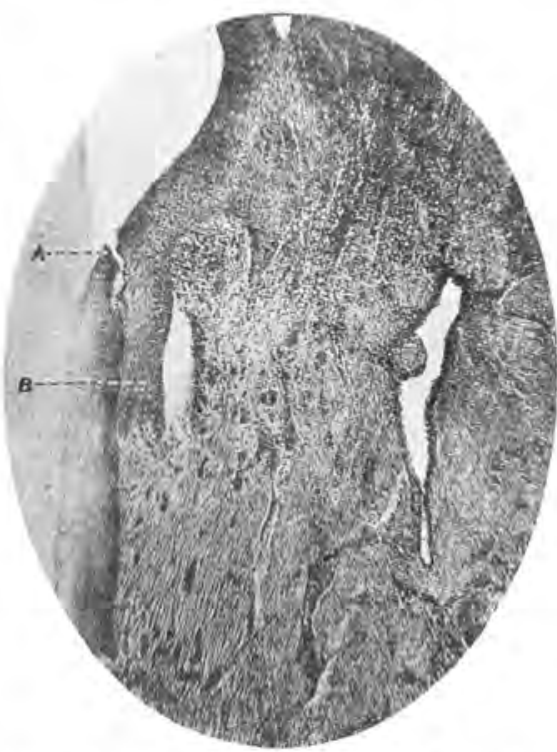

A, Free edge of cementum; B, Oral epithelium of gum tissue. Above $\mathrm{A}$ is a shallow pociset filled with pus during life,-Smilh.

blood supply being received from end capillaries the return flow is blocked by swelling, thus creating a favorable field for bacterial invasion. We find the lingual surfaces of the lower central in. cisors are particularly subject to the accumulation of salivary calculus and it has been the experience of every observing dentist that we have recession of bone and gum on the lingual surfaces of these teeth, accompanied by a com. paratively slight inflammatory disturb. ance with no macroscopic evidence of pus, just a low grade gingivitis. It is also 
true that gingival inflammation and loss of bone does not oecur on the gum margins of patients who have consistently practised oral prophylaxis on the tooth surfaces, so that the tooth surfaces are free from bacterial and mechanical irritants.

That we may more clearly correlate these two facts, namely, that clean tooth surfaces and hard gums prevent gingival inflammation, the author invites your at-
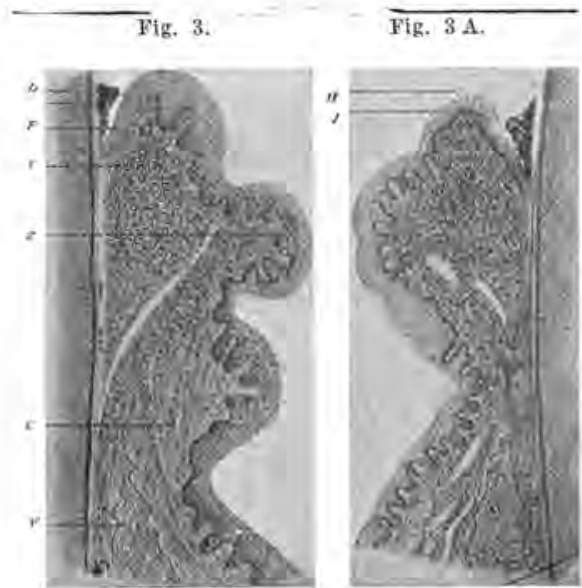

Fig. 3,-d. dentine of the tooth; p, tirtar: $\mathbf{f}$, papillary layer of gum; $\mathrm{z}$, epithelial covering; i, inflitration of the gum with white blood corpuscles; e, healthy part of the gum; $y$, healthy bone. Magnified 80 times.-Zuamensky.

Fig. $3 \mathrm{~A}$-Shows the same parts at a further extenston of the disease. The peeling off of the epithelial covering can be seen (h) ; a wounded surface is being formed (y). Magnified 80 times. - Znamensky.

tention first to a study of a series of stained tooth surfaces in mouths in which no especial care had been taken of the tooth surfaces in the gingival neighborhood, but in which the teeth to the casual observer seemed to be clean.

You will notice from the illustration that the necks of all these teeth and approximal surfaces are black. This black color is due to the application of a solution of iodine and iodides, first suggested to us by Doctor Talbot for treatment of inflamed and irritated tissues and later modified by Doctor Skin- ner for the purpose of revealing to the eye of the observer the bacterial masses upon tooth surfaces.

The question is, what is this material which stands out in such bold relief when treated with disclosing stain? Is it simply muco-plaque material, or is it living bacteria, or both? If it is largely bacterial, what are the most constant forms found on tooth surfaces? The study of hundreds of smears taken from this locality on the tooth surfaces discloses the fact that we have universally present on the tooth surfaces and at the gingival margin, several types of bacteria, and these types do not stain equally well with the same stain. The absolutely universal type which stains best by the Gram method is the streptococcus viridans or the streptococcus salivarus, by others called the streptococcus mites. This organism is absolutely universal and the writer believes it an impossibility to find a human mouth that will not produce this organism. If the Giemse method for staining bacterial smears be followed, one is equally certain to find the presence of fusiform bacillus and spirochaete. The author believes that this member of the streptococeus viridans family just mentioned and the fusiform bacillus and its accompanying ${ }^{\text {* }}$ spirochaete are universally present in all pyorrhea pockets and tooth surfaces. The fact that some observers report one thing and some another does not alter the fact that these bacteria are present. It is merely an evidence of the fact that the individual has not followed proper methods for staining to make their presence evident. If he is dependent on one type of stain, it may be that that particular stain was not suited to bring out the character of growth there present. For instance, the Gram method for staining does not show clearly spirochaete and fusiform bacilli, while the Giemse method for staining will show fusiform

T. 3. Oleary in Dental Cosmos 1910, Vol, 52. page 52 . 
bacilli very clearly while certain other types of bacteria will not stand out clearly. The tooth surface scrapings have been shown to contain almost every sort of bacteria that grows, but the pyorrhea pocket itself has thus far revealed to the author and his associate only two types of organism which seem to be absolutely constant, namely, streptococcus viridans, and the fusiform bacillus with its accompanying spirochaete, staphylococci, pneumococci, proteus, bacillus-coli, subtilus, micrococcus-catarrhalis, and many other bacteria are occasionally found and the entamoeba is present in a certain number of these cases. The author and his associate, Doctor Henrici, have not found the entamoeba to be universally present. It is true that we have found the entamoeba in three-fourths of the cases examined, whereas we find the spirochæte, the fusiform bacillus, and the streptococcus viridans absolutely universal in their presence. In fact in our search for bacteria-free root ends in living teeth, we discovered that it was necessary to actually burn with a cautery the tissues under the gingival margin if we expected to find the root ends free from bacterial growth, and until we did so begin to sterilize by actually burning the lissues beneath the gum margin, we always without exception found the tooth's root infected. Personally, the author believes it to be almost an impossibility to extract a healthy living tooth and find the root free from viridans unless the tooth has been rendered free from bacteria by rubbing the tooth with iodine and by subsequently burning the gingival field with the actual cautery. Even with the above precautions we found living teeth whose roots were infected, showing that the vessels of the peridental membrane form the path thru which root ends are most commonly infected.

The finding by culture of viridans below the level of attachment in the mem- brane also impresses us with the thought that streptococci are the principal organisms of pyorrhea and that amoeba and all other germs are contributing factors only. After having worked this technic out we were able to occasionally extract living healthy teeth and find the deeper tissues sterile. Furthermore, in working along this same line, we find that when the Ionic method of sterilization has been followed out carefully in teeth that previously show$\in d$ areas or rale-iaction by the radiograph and bacterial infections by culture, by practising this careful technic for the exclusion of possible bacteria that have gotten into pyorrhea pockets irom the tooth surfaces and of mouth fiuids that the abscess sack on extraction would be sterile, proving conclusively that it is possible to actually destroy bacterial growth in the tissues of the abscess sack by this electrolytic* method.

The author introduced this bit of experience here because it tends to prove the universal presence of streptococcus viridans in the tissues surrounding the teeth, and, while we all recognize the fact that we may obtain from tooth sur. faces at one time or another almost every known bacterium, we certainly must concede that the bacteria which we find constantly deep in the tissues are the ones logically concerned in the inflammatory disturbances going on in these tis. sues. Some of you may be surprised that the writer does not attach more importance to the presence of entamoeba in pyorrhea pockets and does attach so great importance to bacteria found in the tissues beyond the pockets. Therefore, the writer will break the thread of his discussion by introducing a series of pictures of amoeba to which he invites your attention. Before showing these pictures he desires to say that he welcomed with the greatest enthusiasm

*Rheín Paper, on page 999, Cosmos 1911. 
the possibility of curing pyorrhea alveolaris by destroying amoeba by the simple method recommended by Barrett and Smith of Philadelphia, $\left({ }^{*} 1\right)$, and Bass and Johns of New Orleans, (*2). While the author had occasionally noticed the fact that amoeba are present in the mouth, he had never attached any particular importance to them; in fact, he might state that amoeba have been particularly prolifie in his own mouth for a long time, tho he is apparently free from pyorrhoeal inflammation, so it never occurred to him to connect the amoeba with pyorrhoeal processes in any way. Believing, however, that the work of Barrett and Smith and Bass and Johns deserved full credit, he obtained from the Parke Davis and Company one quart of one per cent emetine chloride and very promptly began its use on a series of pyorrhea cases under his care, following carefully the technic advised by Barrett and Smith in this work, which is daily dosage of one per cent solution of emetine chloride discharged deep into the pockets for a series of six days, then alternating the dose every other day until twelve or fifteen doses had been given, hoping thereby to cure these cases. The writer's experience has been that the injection of emetine chloride has not in a single instance stopt pus flow, tho amoeba have been found to be present in three-fourths of the cases.

Fearing that the emetine chloride solution might be at fault, the author's associate, Doctor Henrici, administered ten cubic centimeters of the chloride to a rabbit which promptly evidenced all the constitutional effects of emetine, subsequently dying. Failing to get satisfactory results or results tending to cure with one per cent solution injected into the pockets, we therefore, obtained fifty grains of eme-

*1-Barrett and Smith, Dental Cosmos, 1914.

*2-Journal American Medical Association. Fob) 13, 1915. tine and commenced a systematic use of emetine hypodermatically, giving as a routine one-half grain per day for three days, then one-half grain every other day for two or three days more, then resting. In no single case has the writer been able to notice freedom from pus flow. One patient, a child of twelve years, who had an exaggerated case of phorrhea having lost all the teeth in the upper jaw but the right cuspid and all the teeth in the lower jaw but the second molars and bicuspids, received in all twenty injections of emetine chlor-

Fig. A

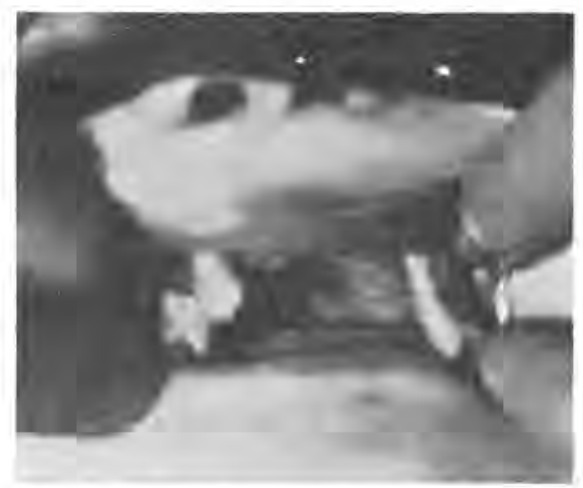

Mouth of twelve year old child suffering pyorrhea, unsuccessfully treated with emetine hydrochloride,

ide into the pockets about these teeth and subsequently two and a half grains emetine chloride hypodermatically. The pus flow has not in any measure been checked as far as the author is able to judge. The gums are inflamed and at a recent public clinic at the mid-winter meeting of the Minneapolis district Dental Society, many men at this clinic were able to see pus from the gums of this patient. (Lantern slide of Figures 4 and $4 \mathrm{~A}$ ). Lantern slides of amoeba (Figures 5 A, 5 B, 5 C, 5 D.)

In this connection, study closely the amoeba found in the following lantern slides. You will notice that the amoeba in these preparations were taken from 
pyorrhea pockets fixt in a saturate solution of bichloride of mercury for a half hour and subsequently stained by the Giemse method. You will note that the ectoplasm and endoplasm stand out sharply. The digestive vacuoles and nuclei show equally well, and you will also note what is infinitely more inter-

Fig. $4 \mathrm{~A}$.

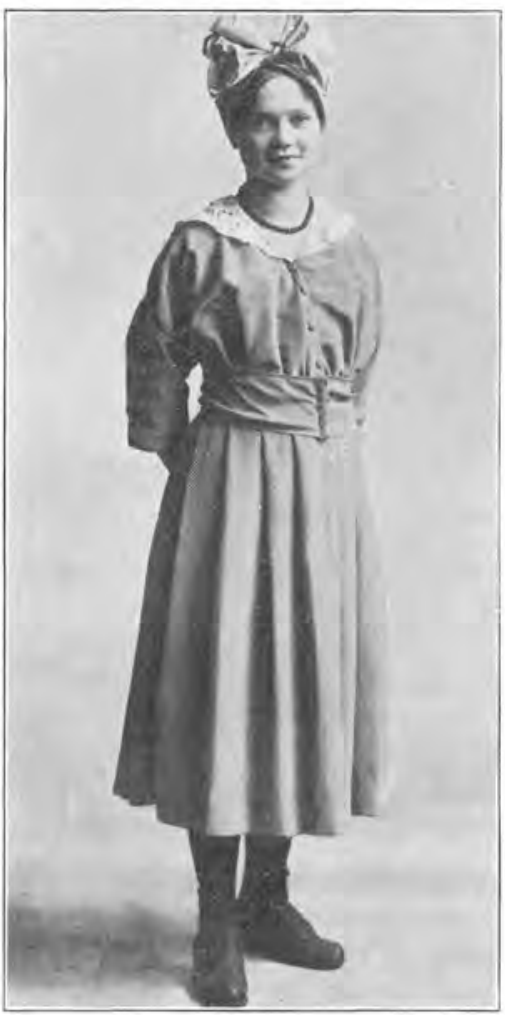

Picture of this healthy, robust looking child.

esting than any other fact about the amoeba so far as the author and his associate, Doctor Henrici, have studied them, that invariably these protozoa contain within their limiting membranes, many bacteria. The author personally has counted seventy micrococci within the protoplasm of a single amoe- ba and almost invariably these amoeba, when properly stained, are shown to be in the process of phagocyting bacteria,

Fig. $5 \mathrm{~A}$.

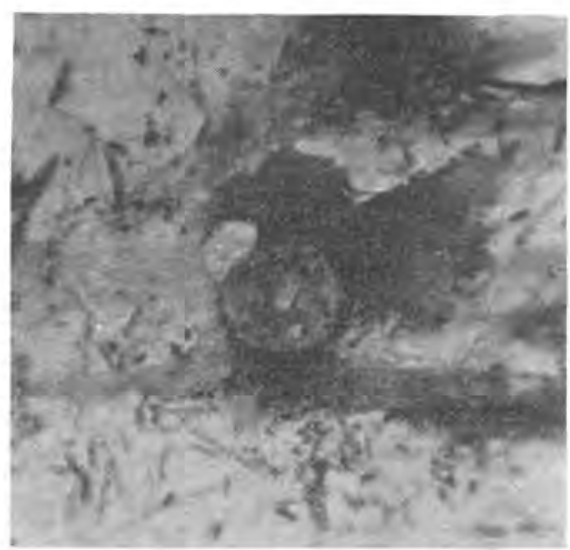

Entamoeba buccalis containing a phagocyted chain of streptococei.

while only about half of them contain within their protoplasm the remnants of other tissues. It is true that a few of the amoeba studied by us seem to con-

Fig. 5 B

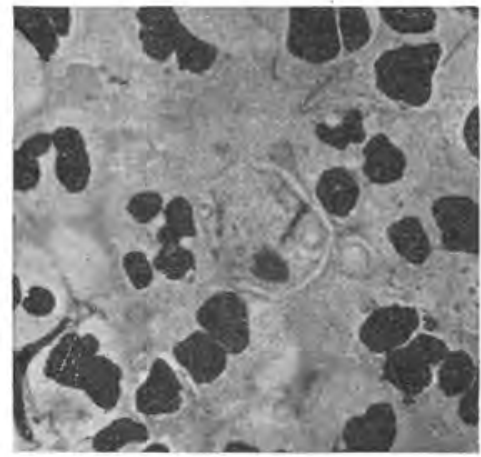

Entamoeba buccalis containing phagocyted fusiform bacilli.

tain the phagocyted nuclei of leucocytes, but the amount of phagocyted material within the limiting mombrane of the amoeba seems to be comparative- 
ly slight when contrasted with the great number of bacteria within their protoplasm.

The thought naturally comes into the

Fig. $5 \mathrm{C}$.

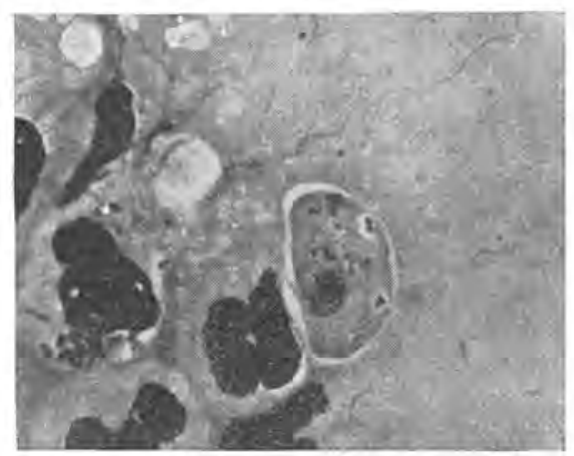

Entamoeba buccalis containing phagocyted diplococeí.

mind of any one who studies this group of pictures, can an organism in the tissues which is so destructive of bacteria

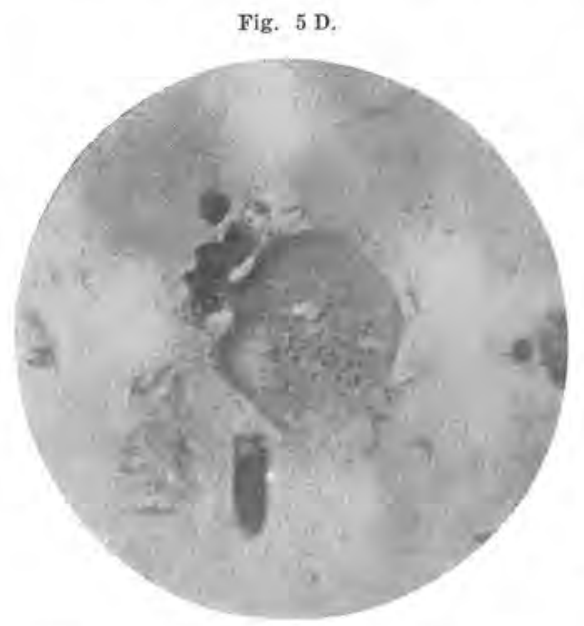

Entamoeba buccalis showing phagocyted cocei.

which we absolutely know to be responsible for many types of secondary inflammation as well as the chief etiological factor in apical abscess. I repeat, can an organism, which is so destructive of bacteria which are harmful to the human body, be accounted unfriendly? If all amoeba are as destructive to bacterial growth as are the ones shown on the screen here, it would seem a better plan to perpetuate their growth rather than to destroy them.

I here invite your attention to a se-

Fig. 6.

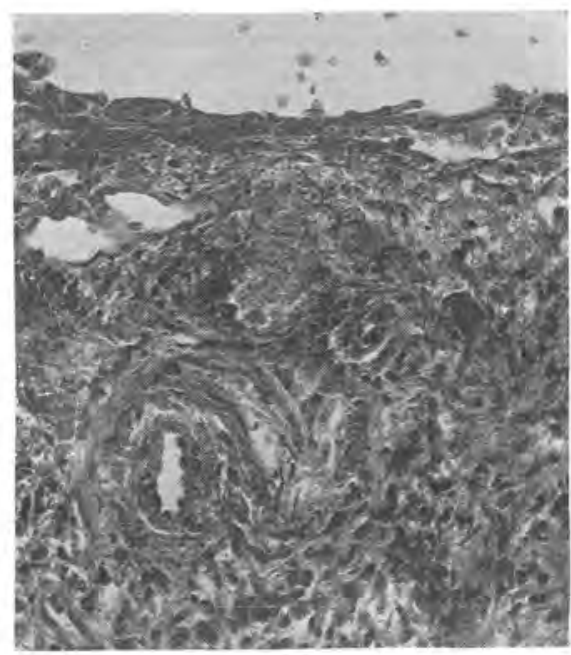

Pyorrhea alveolaris: Ulcerated margin of the gum showing chronic inflammation-no amoeba in the tissues altho many were present in the pus.

ries of lantern slides showing human tissue taken from the sides of human teeth which were exhibiting intense pyorrhoeal inflammation. These tissues were removed with the teeth and hardened, cut, and stained for the express purpose of studying their amoebal content. We find many bacteria on mucous membranes, and the smears from the pockets taken before these tissues were cut away showed a few amoeba and infinite numbers of what proved to be streptococci in culture. A close study of these tissues, however, does not reveal a single amoeba embedded in the tissue. It would seem if amoeba were 
pathogenic that these five cases should show amoeba embedded in the granulation tissues of the bottom of the pockets, but they do not.

In the last two years, we have been encouraged to believe by Hanssen of Christiania $\left({ }^{*} 1\right)$ that there was a specific micro organism responsible for pyorrhoeal infection, designated by him as bacillus pyorrhea, tho the writer has been absolutely unable to find an organism corresponding to the bacillus pyorrhea of Hanissen in pyorrhea pockets. The bacillus subtilus, a common laboratory growth, more nearly fills the morphological and cultural requirements of Hanssen's bacillus than any we have yet been able to find.

The medical and dental professions all over the world have been lead to believe that the amoeba is the responsible factor for all pyorrhoeal processes and that its destruction would be a panacea in the cure of pyorrhea, and the author earnestly hopes tliat this may be true. Nevertheless, his own experience does not lend color to this belief and we are thrown back upon the best teacher we have had in this matter up to the present time, experience, which indicates in no uncertain terms that the gingival margin is the point where pyorrhoeal inflammations begin and that the pyorrhoeal inflammations are wrought by and maintained by the poison proteins $(* 2)$ induced by the presence and destruction of mixed bacterial infections, by the body ferments, and experience has also taught us that clean tooth surfaces preclude gingival infection and that heavy massage of the gums also tends to harden and render more resistant the gingival margins to infection. Experience has further taught us that the logical place to begin the treatment of pyorrhea is the tooth's surface and that the first step in the treat-

*1-Journal of Norweigan Dental Society, 1914.

"2-Victor C. Vaughan's-"Protein Split Product in Relation to Immunity and Disease." ment of any pyorrhea is to demonstrate to the patient and to the operator the relative amount of bacterial growth on the tooth's surface. (Fig. 7) This is important to them both, important to the patient because it directs his attention to those areas of the tooth's surface which need the greatest daily care, and important to the operator because it explains to him the reason for gingival inflammation where often times no other rational cause can be found. Following

Fig. 7 .

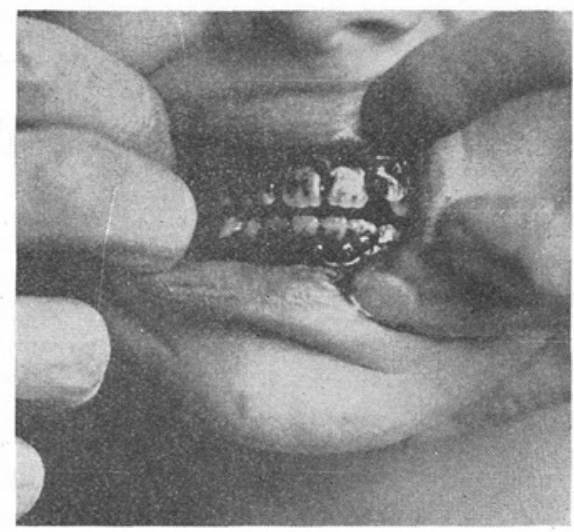

Bacterial masses on tooth's neck.

such demonstration, the next step should be the elimination of all rough areas on the tooth's surface which may retain bacteria on or in the tooth's surfaces, and this is accomplished by applying to them, the type of stones shown on the screen, (Fig. 8) swinging them across the enamel surfaces, thus grinding out the pits that have been induced by the acid formed by the bacteria on these surfaces, getting rid of the bacteria themselves and polishing out the inequalities which serve to retain and foster bacterial growth. After the use of the coarse stones on the tooth surfaces, disclosing stain should be again used to make sure that all pits have been beveled out. After all pits that 
contain bacteria and inequalities have been beveled out, and the application of the disclosing stain reveals nothing, then the tooth surfaces should be thoroly polished with the type of Arkansas stones shown on the screen, and the brilliance increased by the use of Moosehide wheels loaded with jeweler's rouge or with any other abrasive which will give a high degree of brilliance to the enamel.

When the tooth's surface has been properly cared for in this manner, we are then ready to attack root surfaces.

Fig. 8 .

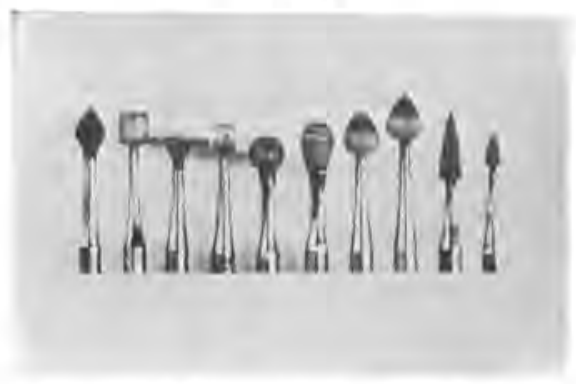

Arkansas and Carborundum stones used to perfect the tooth surfaces.

Before proceeding to a discussion of the pyorrhea pocket, the author desires to discuss for a moment other forms of irritation than that of bacteria which may be responsible for gingival inflammation and will make the statement that any irritant which will induce congestion of the gingival margin favors bactorial infection; e. g. lack of contact point, permitting the bruising of the soft tissues ill fitting regulating appliances, the mechanical irritation of bands, imperfect fillings, malocclusion, thus delivering undue force in some point in certain direction, all of which induce congestion and which in time stimulates osteoclasis and destruction of the bone just as surely as will bacterial growth or the continued application of force by regulating appliances.
There is an additional reason for beginning the treatment of pyorrhoeal in fection on the tooth's surface, the intro. duction of polishing materials into the pockets themselves. Pumice or silex introduced into the pocket must always be an irritant to the tissue cells of the surrounding area. If all polishing and cleansing of the tooth's surface is done before the pockets are disturbed by scalers or planes, we avoid getting polishing material into them.

There may be a specific micro organism which is responsible for pyorrhoeal infection. While we have not proven the streptococcus viridans to be the specific organism, we are thoroly of the opinion and belief that this family of viridans is the chief offender, because of its universal presence in the pockets and in the tissues and because of its definitely proven relationship to dental abscesses and secondary lesions. It is not proven, however, that it is certainly the etiological factor, tho the evidence is slowly accumulating which in the end may be accepted as proof, sufficient to justify our believing it to be the specific factor, for wherever this organism locates in the tissues the result is death of tissue and the question naturally arises, where and how does it enter the tissues? Some authors ("1) would have us believe that it is blood borne and introduced thru the tonsil or from some other area of the body. The anthor does not believe this to be true, however, for the reason that most observers unite in the belief borne out by experience that the streptococcus found in tonsils is haemolytic. Davis $(* 2)$ reports ninety per cent out of 115 cases to be haemolytic. Rosenow's observations confirm the belief that the streptococeus found in tonsils is haemolytic. Dwyer and Gignoux also confirm this

*1-Trich Dental Review 1914. 1912 . 
fact. (*1) In the author's experience with two hundred and fifty cases, only one root tip abscess was proven to be haemolytic, which streptoccocus was found and isolated from a dental abscess in the mouth of a young Hollander by Mr. Gaskill in the early part of the winter of 1914. (*2) With this one exception, no other example of haemolysis was found by Mr. Gaskill and in some two hundred cases in which the streptococcus viridans has been isolated in pure culture by Doctor Henrici, no single example of haemolysis has been observed of a family of streptococci isolated from root tip abscesses or pyorrhea pockets. Gilmore and Moody (*3) reported a short series of cases in which they found haemolysis but the haemolysis was not definitely proven to be due to streptococci as they cultured directly from the infected field to blood agar, and there are other bacteria save haemolytic streptococci which will haemolyse blood, and the hacmolyses reported by them may be and is probably due to the admixture of other bacteria in their cultures. In our own work, our cultures (*4) have invariably been plated out until the bacteria were obtained pure, and I repeat, none of these cultures have been haemolytic. I, therefore, do not believe that the infecting streptococcus in either the dental abscess or the pyorrhea pocket is introduced from the tonsil or the general blood stream, because if it came thru the tonsil path and was deep in the tissues, it certainly should haemolyse, $(* 5)$ We are, therefore, thrown back on the belief that these bacteria are introduced into the tissues by direct continuity of

\footnotetext{
*1-Dwyer \& Gimnoux Bacterial Exam. of Tonsil crypts, from Manhattan eye, ear, and throat reports, Feb. 1912-1913.

*2-Hartzell Dental Bulletin 1914.

* 3-A study alveolar abscess and infected root canals.-Journal A. M. A. December 5 .

4-National Dental Bulletin-Report on Infections of Mouth-Hartzell, Henrici, Leonard.

*5-Barn's-The tonsils, pages 68-69.
}

tissue following down from the tooth neck into the pocket and also that apical abscesses may be planted from bacteria introduced in the same manner or thru the root canals themselves. That it is easily possible for bacteria to enter the peridental structures from the gingival culdesac is a fact. The writer of this paper has repeatedly demonstrated openings in the bottom of the gingival crevice of seemingly healthy teeth and these openings may readily be shown to exist by the use of oxygen under compression which may be allowed to flow from a blunt nosed needle into the gingival crevice. The needle is not to be thrust into the tissues but simply introduced into the gingival crevice, without pressure of the point into the tissues. A stream of oxygen thus directed into the gingival crevice will usually find its way from two or three points at least into the tissues and its movement in the tissues may be noticed for distances of as much as a half an inch or more away from the point of entrance.

The author has repeatedly demonstrated this possibility in the case of his own teeth, the gums of which are healthy, firm, and pink, and has also noted it in a large number of other individuals and he believes that it is thru these openings leading into the deeper structures around the teeth that the bacterial infection of these structures occur. Without doubt, the most valuable work that has yet been done in the gross pathology of inflammation of the tissues contiguous to the teeth is that of Doctor Talbot, (*1) and the author now invites your attention for a few moments to pictures from the work of Talbot. Doubtless, these are familiar to most of you and the writer desires these pictures here in order to illustrate a phase of the treatment of this disease which has always secmed exceedingly important.

\footnotetext{
*1-Talbot-Interstitial Gingivitis.
} 
(Lantern slides of Talbot illustrating lesions of pyorrhea.) You will notice in these (Fig. 9) pictures that the soft tissues are ulcerating, that the peridental membrane has been destroyed to a considerable depth, and that the cemental coat of the root surface is rough and irregular, and that there is no exposed bone showing in the pockets. You will also notice the dense masses of leu-

I.5. 3.

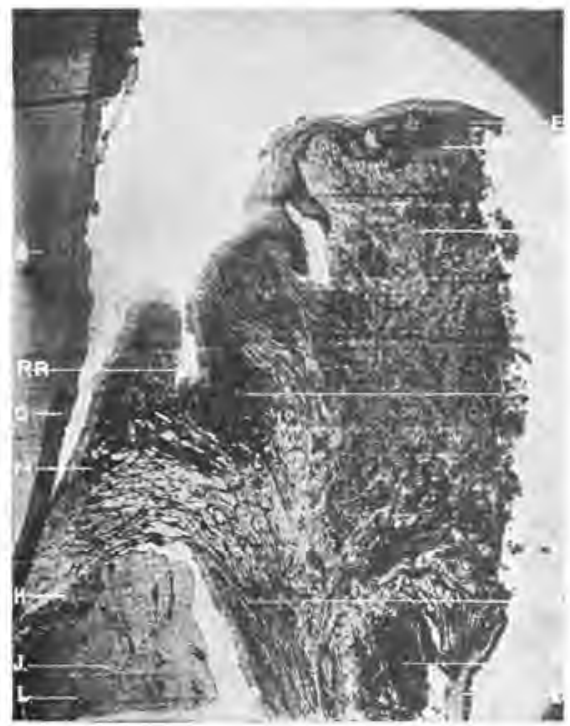

Rough root surface, ulcerating gum margin, and bone of the alveolar process covered by a layer of granulation tissue.-Talbot.

cocytes which predominate in the mass of ulcerating tissue surrounding these roots. The application of the principles of surgery to a study of the lesions of this type would seem to indieate that the first thing to be accomplished would naturally be the removal of the dead, dying, and necrotic tissue which pockets of this type are sure to contain. The enormous mass of leucocytes encompassing this field ought under normal conditions to overcome these infections. These leucocytes are naturally engaged in phagocyting the bacteria growing in these pockets, and there is not the slightest doubt that this wall of leucocytes destroys an enormous amount of bacteria lound growing in these crevices. In addition to the phagocyting of this bacterial mass, the wall of leucocytes acts also as a limiting factor in the spread of bacteria from these foci into the general circulation. However, we all must realize that the leucocytes tho endowed with amaeboid movement cannot and do not readily cross the crevice to the root itself and the bacterial examination of the material of the root itself shows that it is always heavfly loaded with growing organisms. It naturally follows therefore that on account of the rather limited movement of the leucocytes that they only do efficient work in the destruction of bacteria, as bacteria are brought in close contact with them and the tooth's root surface must of necessity receive little or no help from them. Here for a moment study pictures of tooth root surfaces as they appear when the fibre ends are digested out of the tooth's root surface by appropriate media. (Show lantern slides of tooth root surface.) Figs. 10 and 11.) You will note in the study of this second slide which is under a quarter inch subjective that it presents three distinct characters of tissue, namely, this rough surface that has been untouched by instruments which appears on the left. The center of this field shows a great many lacunae and the right side of this same field shows a plane surface in which there are a comparatively few openings. This particular slide was especially prepared to contrast these three conditions.

The author next invites your attention to this picture which shows distinetly the dentine of the tooth structure and outside or overlying this dentine field, you will note the inner layer of the cementum in whỉch you see a great number of lacunae. (Figs. 11 and 12,) 
These lacunae are most numerous near the ends of the dentinal tubuli and di. minish in number as we approach the outer surface of the root. Turning back to the slide which presents the three characters of surface, you will notice that as we closely approach the surface of the root there are comparatively few cemento blasts in the lacunae of the cementum and that there is a consider-

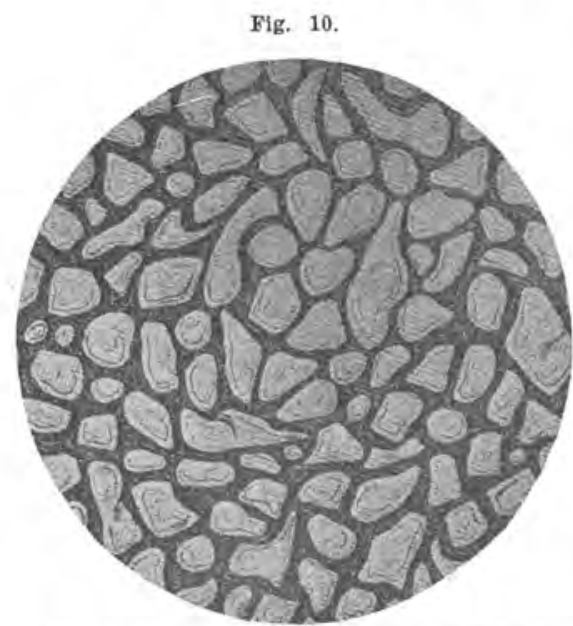

Area of root surface showing its pitted character, the white areas being the stump holes of fibre ends. The dark areas show the more dense walls of the pits, prepared for Dr. Harizell by Doctor Carl R. Lemstrom.

able breadth of tissue in which there are very few or no cementoblasts. I wish you to give particular thought to the morphology and histology of this tissue, as the writer believes it to be infinitely important in the treatment of pyorrhea pockets, because it affords the reason for planing root surfaces and also shows just the depth one dare cut to advantage. Following the general rules of surgery with infected pockets of this type the natural thing would be to curette the ulcerating tissue. As far as the necrotic remains of the soft tissues surrounding the tooth's root is concerned, that is surgically easily accomplished. As for the alveolar process itself, you will notice from a study of Doctor Talbot's pictures that the bony process itself is seldom exposed in the base of

Fig. 11 .

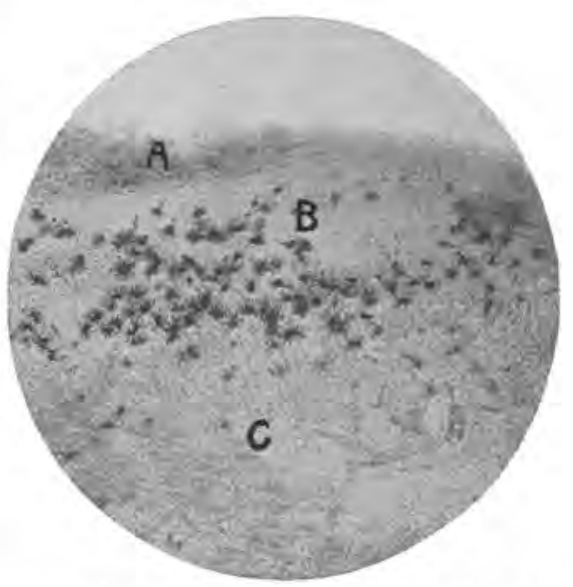

A, Surface correctly planed; B, area of root too deeply planed; $\mathrm{C}$, area of root surface not treated.

the pocket, and that the very deepest portion is densely packed with leucocytes. Therefore, the greater amount

Fig. $11 \mathrm{~A}$,

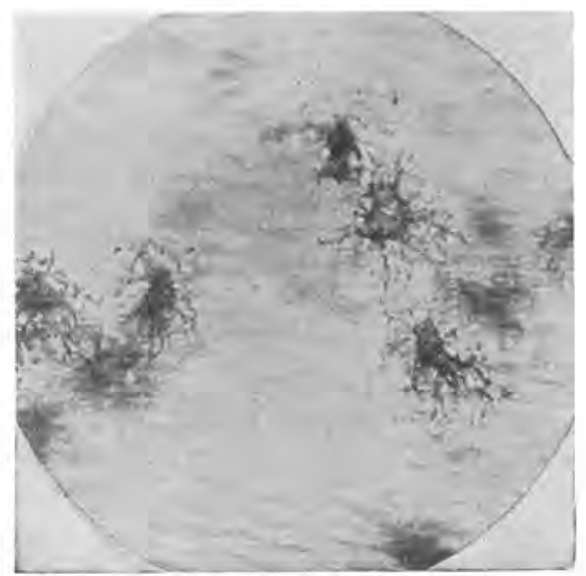

Shows lacunae from center of fleld in Fig. 11.

of materi.1, which is stinking and necrotic, to be removed from these pockets is resident on the root surface itself 
and experience in thousands of cases has taught us that careful removal of serumal deposits in those cases where serumal deposits (Figs. 13 A, 13 B) exist together with the careful removal of necrotic remains of the peridental membrane wonderfully increases the health of the surrounding tissues in a marvelously swift manner. The exact technic of doing this work on the root's surface involves very much more than would appear from a casual study of these tis-

Fig. 12 .

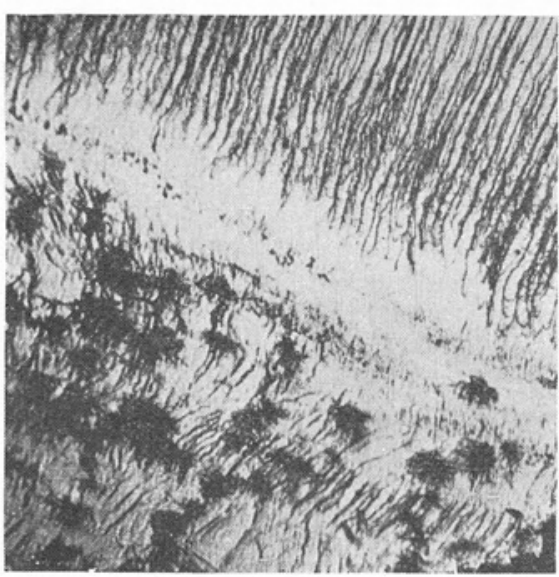

Lacunae near ends of tubuli.

sues. In the first place, the amount of dead necrotic material upon the root surface, while considerable in area, is very shallow in depth and when instruments of any character, planes, files, or scalers are thrust into these pockets, the operator should have in mind the structure of the tissue upon which he operates for the simple reason that if in removing the dead necrotic root surface, the operator carelessly cuts down a sufficient depth to open the lacunae of the cementum, that tho his initial result may point toward health in the improved appearance of the gums and surrounding tissues yet in the end these root surfaces re-infect because the lacunae have been opened and their protoplasm must soon die and become the host of an infinite number of bacteria. Therefore, the surgical treatment of these root surfaces should be done with great care and delicacy and the operator should aim to remove all of the dead necrotic remains and leave a brilliant smooth surface. Such surfaces do not readily re-infect as they have nothing upon them to hold bacteria whereas roots that are scraped deeply

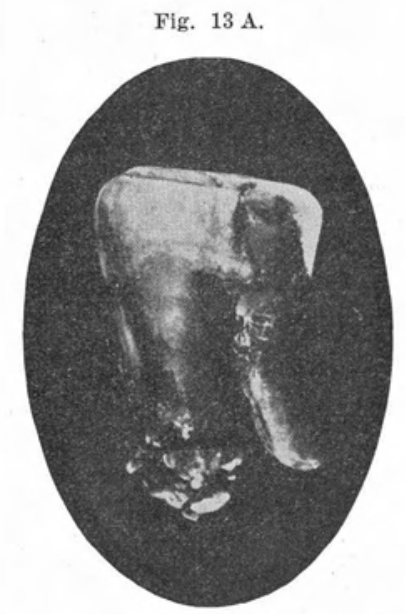

Calculus on root surface. How futile to expect medicines of any kind to benefit a tooth of deposits of this character.

into the cementum will always hold bacteria. The writer believes this fact explains why certain operators who handle the teeth they treat roughly have many recurrences of infection while others who have greater skill and patience have comparatively few recurrences of infection. The moment the dead root surface has been carefully removed, the inpour of new leucocytes is exceedingly rapid because the blood vessels which supplied the bone and peridental membrane have not been entirely destroyed, tho their ends have been blocked by an obliterating endarteritis, which is the specific lesion of pyorrhoeal infection. The operative interference necessary to 
clear the root surface breaks down these endarterial structures and permits of a great outpour of blood into the pyorrhea pocket itself. This bleeding is an excellent thing as it tends to carry out of the pocket infinite numbers of bacteria and also introduces into the pocket fresh arterial blood, which is nature's provisional filler of every wound and a better antiseptic for such wounds than any drug which we could place in them. To briefly describe the character of this type of surgery may be of some interest

Fig. $13 \mathrm{~B}$.

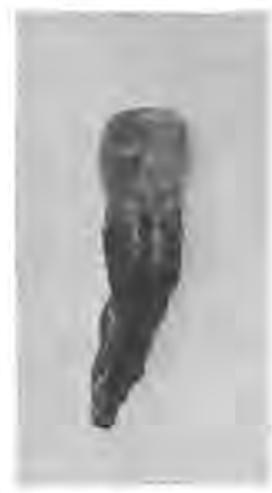

Caleulus on the root surface.

in this connection. It is most easily accomplished with the planes of self-limiting cutting depth. The operator should begin at the neck of the tooth and with short strokes remove the dead material, each stroke going a trifle farther into the pocket until he finally has planed off all the objectionable material to be removed. As the surface cannot be seen the operator must depend on his sense of touch. On root surfaces where there is no deposit, the sharp plane encounters first the dead peridental membrane which evokes a sensation which the author might liken to touching silk velvet, a sort of fleshy feeling. A few strokes with the sharp plane removes this velvety material and brings the plane in contact with the uneven bony surface of the root itself. This produces a sensation entirely different than that produced by the touching of the dead peridental membrane and cannot be well described in words. If, however, the root has upon it serumal calculus, the sensation conveyed to the operator by the plane bit thru the medium of the handle of his instrument and the plane bit, might be comparable to the application of a steel edge to a concrete or stony surface. Upon removal of this stony, gritty material in the one case, or the removal of the velvety dead remains of the peridental membrane in the other, the plane bit rapidly renders the surface smooth and conveys to the operator a sensation comparable to that evoked by rubbing a steel burnisher over polished ivory. In contrast to the sensation evoked by touching dead peridental membrane or calcific deposit, the sensation is so markedly different that after it has once been experienced, the operator can definitely know that he has removed all that should be removed from the root surface. If now the operator, heedless of the fact. that he has reached the hard layer, continued to eut with sharp instruments for a few seconds on this smooth ivory-like surface, which he has been able to produce by the remaval of dead peridental membrane or calculus, the operator will soon find that the instrument will chatter and the sensation will be as vitally different to him as the sensation evoked by bringing a sharp curette over the bony plate of the alveolar process as compared to the sensation evoked by the same curette when used in the spongiosum of the loose areolar bone inside of the bony plate. It naturally follows that the fewer lacunae opened, the less subsequent re-infection. As the operator approaches the bottom of the pocket he will encounter granulation tissue. This grazulation tissue is a mass of leucocytes which interposes between the bottom of the pocket and 
living tissue beyond and serves to protect the living tissue beyond from further incursions of bacteria and also surrounds the obliterated ends of the vessels which fed the tissue. It is an advantage to press the plane bit into this mass of leucocytes in order that every bit of diseased root surface may be removed and also to break up the granulating wall and open the obliterated ends of the vessels in this locality. The action of the plane bit will naturally withdraw from the pocket most of the planed

Fig. 14 A.

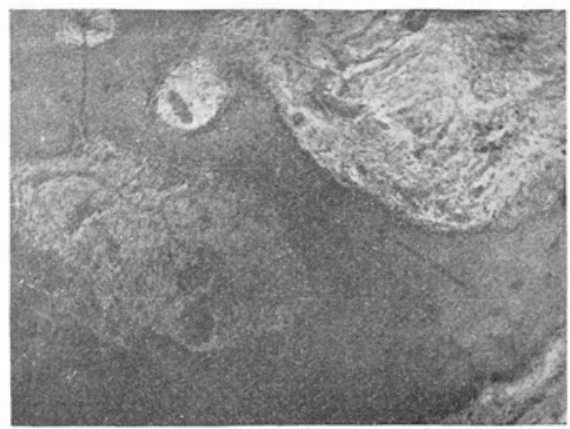

Spongy bone growing in glass tube.-Macewen.

off material. If the operator is in doubt as to whether the pocket is free from loose material, it is wise to syringe the pocket with Ringer's solution or normal salt solution.

Assuming always that the diagnosis has been properly made and the advisability for treatment of any given case determined and that the work has gone forward to the removal of the diseased root surface, the next thought which confronts us is the possible regeneration of bony process to compensate for that which has been lost. The regeneration of bone of course precedes from living bone. This has been well proven by the work of Macewen (*1) of Glasgow, (Figures $14 \mathrm{~A}$ and $14 \mathrm{~B}$ ) whose classical

\footnotetext{
*1-Macewen-“The Growth of Bone.”
}

experiments in this direction must ever be remembered. He was able to remove portions of long bones in dogs and interposed between the cut ends of these bones small glass tubes which glass tubes filled up with new bone in from four to six weeks, the outpour coming from the cancellus bone cells of the ends of long bone and not from the periosteum. Accepting the fact that new bone (Figures $15 \mathrm{~A}$ and $15 \mathrm{~B}$ ) comes from preexisting bone cells, can we do (Fig. 16) anything which will tend to repair the

Fig. 14 B.

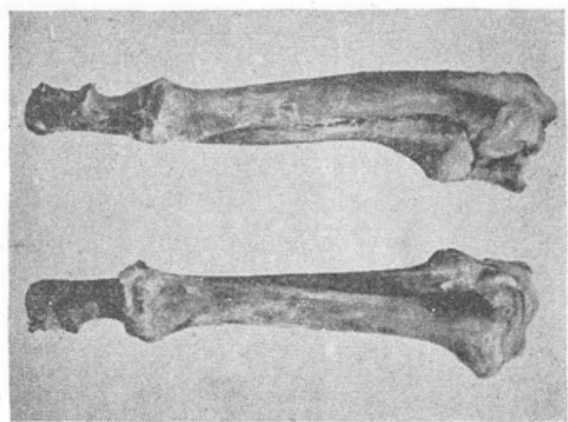

Transplanted sections showing perfect union.

loss of bone already suffered? The writer believes to a limited extent that we can, by simply following the laws of general surgery in relation to bone growth. Therefore, our need is freshly opened bone cells, absence of general infection, absence of pressure, and rest. Presuming the pyorrhea pocket and root surface to be as surgically clean as they can be gotten, our next step is to reach into the process with a fine sharp instrument and lightly stir or roughen up the process edge. This stimulates the bone cells to an outpour of callus. If the pocket be filled with sterile blood clot, the new bone cells pour out and in some instances actually pile up against the root surfaces never in great amount because the pressure of surrounding tissue limits the amount of outpour, be- 
cause we begin to have shrinkage of the overlying soft tissue as soon as we remove the irritating debris of the root surface, which shrinkage of the gum limits the outpour of bone from the thin process

Fig. $15 \mathrm{~A}$.

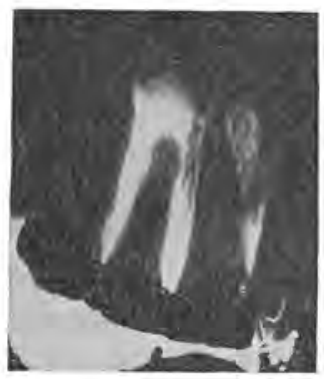

The implanted tooth in socket from which $13 \mathrm{~B}$ was removed.

edge. The subsequent entrance of bacteria from the tooth's surface and saliva into the pocket also tends to limit the bony outpour. Movement of the teeth themselves also tends to limit the bony outpour. If, however, the teeth are made

Fig. $15 \mathrm{~B}$.

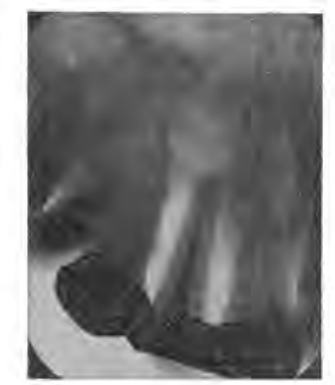

New bone around implanted tooth one year later.

rigid by a splint, the root surface clean, the process edge lightly curetted, the pocket filled with blood and protected from infection, we can confidently expect a certain amount of bony outpour from the process edge. Just in proportion as we can maintain these sensible requirements will we be able to gain bone. Some men deny the possibility of regeneration of bone of the jaws. The author draws your attention to a lantern slide in a case first operated by Doctor R. E. Farr, of Minneapolis, wherein he removed an inch and a half of the man. dible for the removal of a malignant growth. The writer subsequently placed a splint of iridio-platinum wire connected with the left cuspid and laterals and the right second and third molars. A radiograph of this jaw some two years after the splint was put in position

Fig. 16 .

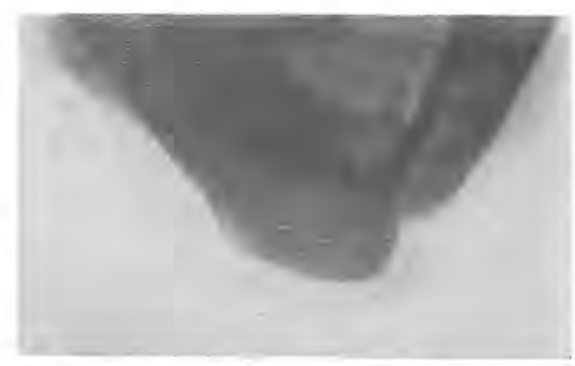

Radiograph of Dr. Farr's case showing complete repair of mandible from whence one inch had been removed. An iridio platinum splint placed by the writer to hold the cut ends apart can be seen in the picture.

shows that the bone reached across from the left to the right side and that the bony continuity of the mandible has again been restored.

The writer also wishes to show in this connection, a picture which illustrates the ability of the process to pour out new bone around and against the teeth. This particular picture was obtained from the late Doctor Fletcher with whom the writer of this article had much in common. Its origin was briefly as follows: (Figures $17 \mathrm{~A}, 17 \mathrm{~B}, 17 \mathrm{C}$ ).

A patient had applied to a dentist for the removal of a lower bicuspid tooth. The dentist had attempted its extraction and had broken the tooth off well below the gum level and had then left this tooth in position. The gum had healed 
and the patient was none the wiser. Subsequent disturbance in that locality caused the patient to apply to Doctor Fletcher, who discovered that this root still remained in the socket. $\mathrm{He}$ cut down upon and uncovered this root and discovered that the alveolar process seemed to cover it. He extracted the

Fig. $17 \mathrm{~A}$.

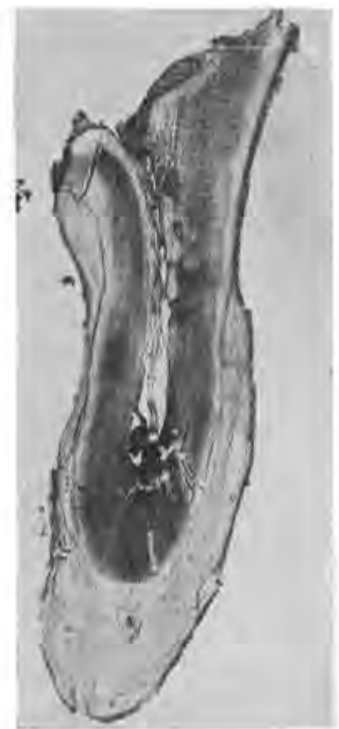

Extracted tooth with new bone in pulp chamber. Fletcher's case.

root and subsequently had sections made, a picture of which I throw upon the screen, showing new bone deposited on the broken root end and pulp chamber of this tooth. A second picture shows an area of alveolar process photographed from the center of this chamber. While we all recognize the fact that the alveolar process is not a favorable field for regeneration, these examples show conclusively that if we can maintain our case under proper conditions that we certainly may expect a small amount of new bone at least in many cases. That bony outpour does frequently happen in the experience of every man who treats pyorrhea is proven by the fact that teeth that have been denuded of a large amount of bony support subsequently treated successfully become more rigid comparatively speaking than healthy teeth that have never undergone pyorrhoeal inflammation. The explanation of this fact is that a small amount of new bone has been poured out and piled up directly against the

Fig. $17 \mathrm{~B}$.

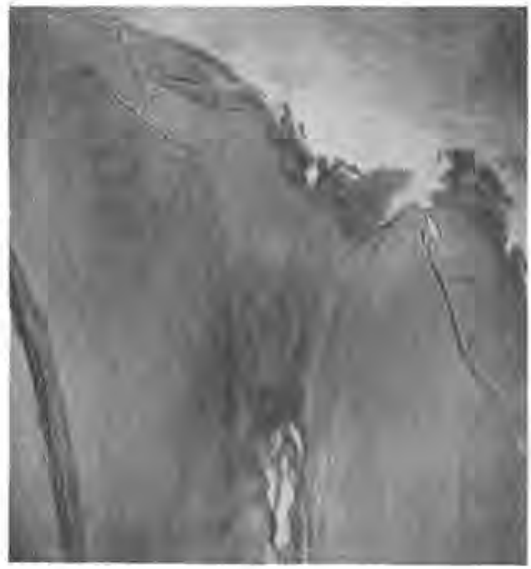

Pulp chamber of $17 \mathrm{~A}$ showing new bone.

root surfaces, and, having no elastic peridental membrane interposed between the root surface and the new bone, you have an analogy to ankylosis which occurs about implanted teeth. Presuming that this treatment of a case has been successfully carried to this point, is there anything further the operator may do to enhance regeneration? The author believes there is, and that the next step should be to protect the sterile blood clot in the pockets from the incursion of bacteria from the saliva and the tooth's surface. The bacterial growth is constantly recurring on tooth surfaces, and its inhibition or prevention must necessitate a constant battle to the end of life. Immediately, following the surgical treatment, the application of 
some substance which will seal the pockets, shut and protect them from bacterial incursion is advisable. Many substances have been recommended for this purpose. The most valuable one of which the writer has knowledge is the following:

Merck's beechwood creosote saturated with all the iodine it will dissolve.

This makes a heavy black oily mixture

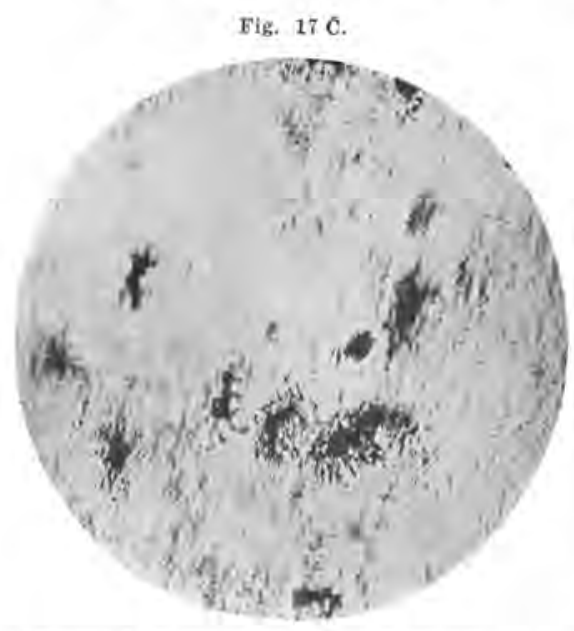

Lacunte from center of $17 \mathrm{~B}$. Fleteher's case.

and is to be applied to the gum edge and to the neck from one-eighth to a quarter of an inch to the gum edge and reaching up on the tooth neck to the enamel of the crown. Carefully preventing the saliva from touching this after it has been placed, we immediately apply a second coat composed of glycerine in which we have incorporated all the tannic acid that it can be made to dissolve. Thus the tannic acid most completely covers the first coat of creosote-iodine, and, if it is placed before moisture comes in contact with the creosote-iodine, the two solutions seem to unite and form a dense, tough, black brown material which will cling to the tissues from twenty-four to forty-eight hours, protecting the gingival margin from a bacterial incursion for that period of time. If thought advisable by the operator, the coating may be repeated every other day for several days, thus maintaining a condition of approximate asepsis. The author might state in passing that the application of this double coating to sutured incisions in the mouth protect these edges from infection and renders a wound much more comfortable than otherwise would be the case. It may also be applied to the cut edges where third molars have been uncovered by removing the gum. As soon as the bleeding has been checked by the application of hot packs, the cut surfaces may be coated with this heavy iodine solution followed by the tannic acid glycerine solution and the cut surface will be protected from infection and also from the irritation of the movement of the tongue. The method of carrying on pyorrhea treatment should follow certain definite lines and the judgment of the operator should be governed by the condition of the patient. In cases where we have only mild gingival inflammations and shallow pockets, the work may be rapidly accomplished without hurt to the patient. On the contrary, if the patient be subnormal in resistance, the pockets deep and heavily loaded with organisms, then the operation should be continued over a period of weeks involving only two or three teeth at each treatment. There are two reasons for this course. The first is that if the limiting wall of granulation tissue so beautifully shown to be present in all these pockets by the pathological work of Talbot and others, I repest, if the limiting wall of granulation tissue be broken down rapidly, an enormous inoculation of the patient occurs at once, and this enormous inoculation may result in an acute disturbance, wherein metastatic transfer plays an important part, and result in a myocarditis, endocarditis, joint infection, or acute abscesses, or chronic infection of the kidney.

The author has learned by bitter expe- 
rience in the treatment of actual cases that haste in the treatment of these subnormal cases is exceedingly dangerous and subversive to the best interests of the patient, as he has noticed acute joint infection and even pneumonia to result in rapid sequence from, as he believes, over-inoculation from the tearing down of the limiting wall of the granulation tissue which must of necessity be broken down in the rapid treatment of these cases. A second reason for slow treatment by easy stages of these pyorrhea cases lies in the fact, that if the dosage of bacteria be gradually increased from

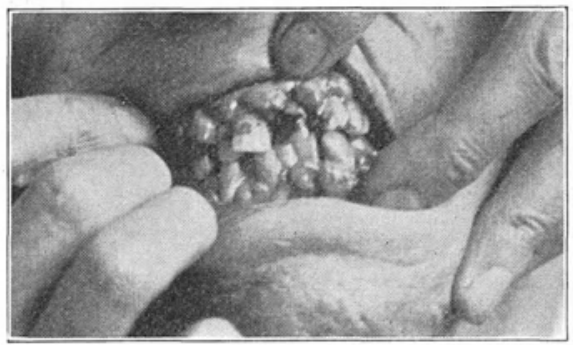

Before Treatment.

time to time, the ability of the blood stream to overcome the recurring doses of bacteria which of necessity must be forced into the blood stream by the surgical treatment, gradually raises the immunity of the individual so that at the end of a period of a month or six weeks' treatment of a case of this type, the patient's general resistance is steadily raised, appetite, ambition, and general well-being enhanced. In fact, treatment so carried on has all the good advantages of a polyvalent vaccine and none of its disadvantages. The post-operative care of an individual deserves also careful consideration.

\section{MASSAGE-WHEN AND WHY ADMIN- ISTERED.}

Massage of the gum margins should not be commenced following operative treatment until all outpour of bony callus from the process edge has ceased for the simple reason that massage tends to shrink and toughen the gum tissue and prevents further bony callus being poured out. As soon as the operator is satisfied with the amount of bony callus which he has gained, he may then begin massage. This massage should be

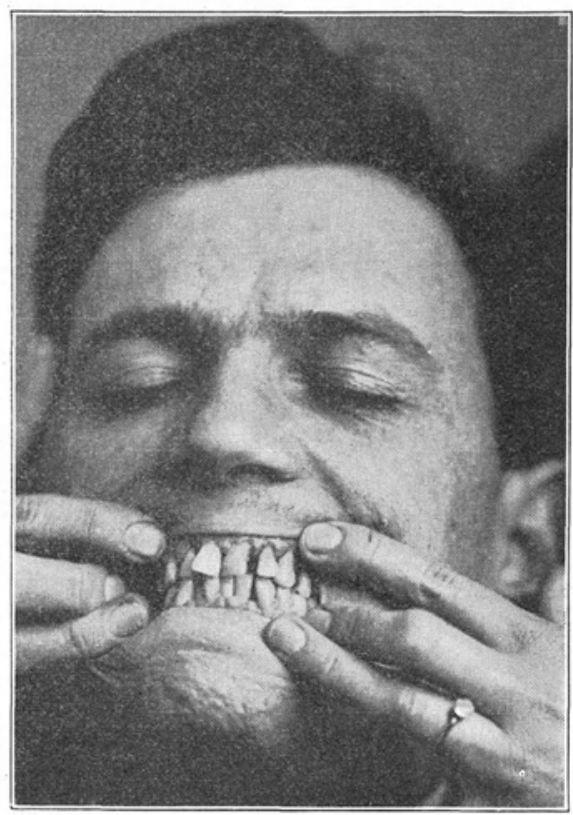

After Treatment.

Blood pressure fell from 150 to 120 . No treatment and no emetine or other drug was administered, showing clearly that it is possible to bring about great changes in the treatment of Pyorrhea without drugs by efficient surgery only.

suited to the gum tissue. Perhaps the best method of massaging the gums is with the cut end of the short heavy cotton roll. The dry cotton roll clings to the gum tissue and when held in a haemostat of a kuroris is easy of use by the patient. Its advantages are briefly that it expels the stagnant fluid from an oedematous gum margin and permits inflow of fresh arterial blood. The act of massaging the gum margin drives into the circulation any bacteria that may 
have gained access into the tissue and places them where the leucocytes of the general blood stream will rapidly phagocyte them. General massage is in itself a kind of auto-vaccination and drives the enemy out of the field of action where it is more easily conquered and replaces the enemy in the field of action with new soldiers from the blood stream with the added benefit that the epithelial coat and connective tissue composing the gum is caused to grow more firm and dense and resistant, thus fortifying the gum margin from further infection by enhancing a more perfect structure and locking the door to further infection. The weekly use by the patient of the disclosing stain to guide him in his daily mouth hygiene has a double benefit. The solution now used by most men for disclosing purposes is that mentioned in the beginning of the article, and, tho many times published by those who use it, the writer will give its composition once more for the benefit of the younger men who do not happen to be acquainted with it: Iodine, fifty grains, zinc iodide and potassium iodide each fifteen grains, water and glycerine each four drams, making a total liquid bulk of one ounce with a total bulk of eighty grains of solid material incorporated therein. This should be very carefully triturated and may be sponged on the soft tissues and tooth surfaces without harm occasionally. The author believes it is too concentrated for daily use. Its double benefit accrues from the fact that it destroys instantly the vitality of the bacteria with which it comes in contact and also brings into view the fields where they grow in greatest number undisturbed, thus guiding the patient to a more careful effort in the daily hygiene of the mouth.

Daily prophylaxis by the patient is the greatest safeguard against reinfection and the monthly prophylaxis treatment by the dentist is the best guide to the patient's endeavor. Treatment by the dentist should be very carefully done and not left to careless hands. In this prophylaxis work the injury of the gum should be distinctly avoided as well as injury of the enamel surface and exposed cementum. It is possible to conceive that too vigorous or ill-advised use of the polishing material on either the enamel or dentine surfaces can do these tissues great harm, even destroying or cutting grooves in them, and has given ground for criticism by many conservative men. This phase of treatment is so well understood that the author deems it unnecessary to discuss it under a special head.

There is, however, one more thought regarding this matter which appeals to the writer as a vast importance, and that is the question of so guiding the daily life of a patient in the matter of food and diet that the oral cavity as well as the balance of the body increases its immunity to all types of infection. The work of Doctor Pickerel and Professor Gies of Columbia in this direction should be read and understood by every one of us. The work at present being carried forward by one of our own number, Professor Bunting of the University of Michigan also should bear fruit in this direction. The net result of the work of Professors G. V. Black (*1), Pickerel $(* 2)$, and Gies $(* 3)$, has been to indicate to us that we can accomplish an enormous amount for our patients by first limiting diet to exactly what we need for our daily regeneration, thus avoiding excessive deposits of calculus, ingesting those foods which tend to produce a salivary fluid inimical to the growth of bacteria, and the use of such fluids and mouth washes which will promote a normal salivary outpour. This to the mind, of the writer is an enor-

\footnotetext{
*1-Black, in Dental Review 1913.

*2-Pickerel on Decay.

*3-Gies' research report, Joumal of Allied Societies.
} 
mously valuable contribution and should never be lost sight of.

The title and scope of this paper does not admit of a lengthy discussion of these phases of the treatment of pyorrhea and prophylaxis, and the author feels in closing his paper, that in order not to create the idea in the minds of this audience that he believes surgicai treatment of pyorrhea is the Alpha and Omega of the subject, it should be stated that the powers that make for immunity should be always carefully considered.

I thank you for your kind invitation to appear before you and for the delightful courtesies that $I$ have received at your hands. 